\title{
Minimal invasive Versorgung von Verletzungen der Brust- und Lendenwirbelsäule unter thorakoskopischer Kontrolle
}

\author{
V. Bühren, R. Beisse, M. Potulski
}

\section{Zusammenfassung}

Über einen Zeitraum von 4 Jahren wurden 300 konsekutive Verletzungen der Brust- und Lendenwirbelsäule unter thorakoskopischer Kontrolle versorgt. Der endoskopisch gesteuerte Eingriff umfasste dabei die Resektion verletzter Bandscheiben und Wirbelkörperanteile, ggf. die spinale Dekompression unter Hinterkantenresektion, den Aufbau der ventralen Säule mittels trikortikalem Beckenkammspan oder alternativ mit Synexkorb, sowie die winkelstabile Fixierung mit einer Plattenspondylodese. Die Analyse der Serie zeigt eine niedrige Komplikationsrate intraoperativ mit einer im Vergleich zum offenen Verfahren deutlich geringeren postoperativen Morbidität der Patienten.

Die anfänglich als Reservemethodik angesehene endoskopische Versorgung der Wirbelsäule ist nunmehr zur ausschließlich angewandten Rou- tineoperation geworden. Die Erfahrungen aus anderen Zentren, die derzeit auf die endoskopische Methodik umsteigen, lassen erwarten, dass binnen weniger Jahre die endoskopische Operationstechnik das offene ventrale Verfahren ersetzen wird. Wesentlicher Faktor für diese Prognose ist zunächst die Besonderheit des Thoraxraumes, der keinerlei expandierende Maßnahmen benötigt, um das Operationsfeld freizuhalten. Die operativen Manipulationen gestalten sich gegenüber den Bedingungen im Bauchraum wesentlich einfacher.

Wie schon für die arthroskopischen und laporoskopischen Eingriffe gezeigt, ist die Zugangsmorbidität dramatisch verringert. Durch die veränderten operativen Möglichkeiten werden sich das Indikationsspektrum und die Durchführung der Wirbelsäulenchirurgie in Zukunft wesentlich verändern.

\section{Einleitung}

Auch für Frakturversorgungen nach dem Prinzip der offenen Reposition und internen Osteosynthese (ORIF $=$ Open reduction, interned fixation) werden zunehmend minimal invasive Zugangswege bevorzugt. An der Wirbelsäule können die aufwendigen offenen Verfahren mittlerweile gleichwertig durch endoskopisch gestützte Methoden ersetzt werden.

Seit 1996 wurden an der BG-Unfallklinik Murnau 300 Patienten nach akuten Verletzungen im Brust- und Lendenwirbelsäulenberich unter thorakoskopischer

OP-JOURNAL 2000; 16: 244-250

(c) Georg Thieme Verlag Stuttgart · New York
Kontrolle operiert. Im folgenden werden Indikationsspektrum, Operationstechnik, Ergebnisse und Komplikationsmöglichkeiten dieser jungen Technik analysiert und kritisch diskutiert.

\section{Historische Entwicklung}

Der Wandel von der konservativen zur operativen Therapie in der Frakturbehandlung hat als letzten Skelettabschnitt in den vergangenen 20 Jahren auch die Wirbelsäule erfasst. Für alle anatomischen Regionen und speziellen Verletzungsformen existieren mittlerweile definierte Zugangswege und Instrumentationen, um eine operative Behandlung bei neurologischen Ausfällen, Instabilitäten oder Fehlstellungen durchführen zu können $[4,10]$.
Im gleichen Zeitraum vollzog sich in der gesamten Chirurgie ein Wandel bezüglich der Operationsmethoden. Große und aufwendige operative Zugänge wurden wegen der Nachteile mit verbleibender oft lebenslanger Morbidität systematisch optimiert. Kleinste Zugänge unter Einsatz optischer Sehhilfen und spezialisierter Instrumente werden heute unter dem Begriff der minimal invasiven Chirurgie zusammengefasst. Für präformierte Körperhöhlen, wie beispielsweise für die großen Gelenke, die Bauchhöhle und den Brustkorb ergab sich mit der Perfektionierung digitaler Kleinstkameras die Möglichkeit zur endoskopisch gesteuerten und videoskopisch visualisierten Operation.

Erste endoskopische Eingriffe in der Brusthöhle wurden schon 1910 unter Verwendung eines Zystoskops vorgenommen [6]. In den Folgejahren wurde diese Thorakoskopie nicht zur diagnostisch, sondern auch therapeutisch zur Lösung von tuberkulösen Pleuraverwachsungen und zur Durchführung der thorakalen Sympatektomie eingesetzt. Mit der Entwicklung leistungsstarker Optiken, der Kaltlichttechnologie und der Mikrochiptechnik wurden seit Anfang der 90er Jahre zahlreiche klassische Eingriffe der Thoraxchirurgie wie Lungenteilresektionen und Perikardektomien endoskopisch kontrolliert durchgeführt.

1993 berichtet Mack erstmalig über den Einsatz der Thorakoskopie auch bei Erkrankungen der Brustwirbelsäule [7]. Seit 1994 beschreiben verschiedene Autoren Eingriffe an der Wirbelsäule bei degenerativen und metastatischen Erkrankungen. 1995 wird erstmals die systematische Anwendung bei Verletzungen der Brustwirbelsäule berichtet [9]. 1997 wird dann die Komplettversorgung von Wirbelsäulenverletzungen mittels Resektion der zerstörten Gewebe, Dekompression des Spinalkanals, Wiederherstellung der vorderen Säule und Retention mittels Plattenspondylodese beschrieben [5]. In- 
zwischen existieren detaillierte Angaben zur Technik und Instrumentierung der verschiedenen Wirbelsäulenabschnitte $[2,3]$.

\section{Offene versus minimal invasive Verfahren}

Die Rumpfwirbelsäule liegt zentral eingebettet im Körper, dabei dorsal von kräftiger Muskulatur und ventral von lebenswichtigen Organen und Leitungsbahnen überdeckt. Aufgrund des relativ großen Abstandes von der Körperoberfläche wird für ein offenes Vorgehen ein großes Operationsfeld zur Aufnahme der Hände des Operateurs unter Verwendung herkömmlicher Instrumente und Implantate benötigt. Dieses große Operationsfeld in der Tiefe mündet im Sinne eines klassischen konvergierenden Operationstrichters in einem großen Hautschnitt. Dieser Schnitt kann bei kurzstreckigen monosegmentalen Versorgungen unter Verzicht auf ventrale Instrumentierungen deutlich reduziert werden. Eine weitere Minimierung ist durch Verwendung von Sperrersystemen möglich, deren Einsatz allerdings deutliche Limitierungen durch mangelnde Visualisierung und Übersicht des eigentlichen Operationsfeldes erfährt.

Die extremste Minimierung des eigentlichen Hautschnitts ist unter Nutzung endoskopischer Techniken möglich, die mit der Entwicklung lichtstarker Optiken, der Kaltlichttechnologie und der Mikrochiptechnik eine umfassende Visualisierung für das gesamte Operationsteam in Videotechnik ermöglicht.

Die Aufteilung der benötigten Operationsinstrumente auf mehrere Portale bringt nochmals eine Reduktion der Länge der Einzelschnitte mit sich.

Im Bereich des thorakalen Raumes ergeben sich für die Verwendung der Endoskopie besondere Vorteile, da die Aufspannung der Weichteile durch den Rippenkäfig die Verwendung von Gas unter Überdruck verzichtbar macht. Wesentliche typische Nachteile endoskopischer Techniken im Bauchraum und im Retroperitoneum mit Druckverlusten während der Operation, systemischen Folgeerscheinungen, erschwertem Absaugen von Blutungen, Gasembolie etc. ergeben sich im Thorax somit nicht.

Die Übersicht in der Thoraxhöhle ist gegenüber offenen Verfahren deutlich erweitert, die Bildqualität bei Nutzung moderner Technologie bestechend. Diffizile
Verfahren wie die Hinterkantenresektion unter Kontrolle der Dura lassen sich vergleichbar einer Operation unter dem Mikroskop durchführen.

Langstreckige Verletzungszonen und Etagenverletzungen können durch Umsetzen der Instrumente in ihren Portalen oder durch Schaffen von nur 1 oder 2 Zusatzportalen umfassend versorgt werden.

Wesentlich für die endoskopische Technik ist, dass im Gegensatz zur Verwendung von herkömmlichen oder minimal invasiven Rippensperrern kein permanenter Druck auf die Rippen und die interkostalen Leitungsbahnen ausgeübt wird. Für die thorakoskopischen Instrumente ist die Weite des Zwischenrippenraums völlig ausreichend und braucht niemals durch Resektionen oder Durchtrennungen der Rippen erweitert zu werden.

Neben den offensichtlich besseren kosmetischen Ergebnissen ist die postoperative Schmerzreduktion anhand der verkürzten Nachbeatmungszeit und des verminderten Schmerzmittelverbrauchs objektivierbar. Für den langjährig früher in der konventionellen Technik tätigen Wirbelsäulenchirurgen belegen die subjektiven Angaben der thorakoskopisch operierten Patienten im Vergleich zu den offenen Thorakotomien die Vorteile bezüglich der Zugangsmorbidität eindeutig.

\section{Potential der endoskopischen Technik}

Die akute Versorgung von Wirbelsäulenverletzungen beinhaltet prinzipiell $4 \mathrm{Be}$ handlungsschritte:

1. Reposition der Fehlstellung

2. Dekompression des Spinalkanals

3. Rekonstruktion der vorderen Säule

4. Retention zur Sicherung einer stabilen Ausheilung

In der Praxis ist mittels der thorakoskopischen Technik nahezu die gesamte Brustwirbelsäule und der obere Bereich der Lendenwirbelsäule von Th 3 bis L 3 erreichbar.

Der traumatologisch hochrelevante thorakolumbale Wirbelsäulenabschnitt Th 11 bis L2 , der mit größter Häufigkeit betroffen ist, wird mittels eines für die thorakoskopische Technik entwickelten Zwerchfellsplittings erreicht [1]. Dabei wird ausgenutzt, dass die hinteren äußeren Recessi der Pleurahöhle bei medialer lotrechter Projektion die Lendenwirbel- säule in Höhe L3 erreichen. Aus dem indikatorischen Spektrum der thorakoskopischen Versorgung fallen somit nur die selteneren Frakturen der unteren LWS sowie die Verletzungen am thorakozervikalen Übergang mit ihrer besonderen Problematik heraus.

\section{Reposition}

Für die Formwiederherstellung stehen zunächst die üblichen äußeren Repositionsmanöver unter Zug und Lordosierung zur Verfügung. An der Brustwirbelsäule kann durch Druck auf die Dornfortsätze auch intraoperativ oft eine suffiziente Wiederaufrichtung erreicht werden. Direkt instrumentell ergeben sich limitierte Repositionsmöglichkeiten durch Aufspreizung mittels spezieller Zangen nach dem Outriggerprinzip, die über die Plattenhalteschrauben am Wirbelkörper befestigt sind. Eine endgradige Aufrichtung kann auch durch Einsatz aufspreizbarer Wirbelkörperinterponate wie dem Synex-Korb erreicht werden.

Für die häufigen thorakolumbalen Verletzungen bevorzugen wir in der klinischen Praxis die Akutreposition mittels Fixateur intern über dorsalen Zugang. Diese Versorgungsnotwendigkeit ergibt sich bei neurologischen Ausfällen als Notfallindikation zur sofortigen Dekompression des Spinalkanals. Bei B- und C-Verletzungen in der AO-Klassifikation besteht zudem die Notwendigkeit einer auch dorsal eingesetzten zuggurtenden Instrumentierung (Abb.1)

Nur bei per CT gesichertem Frakturtypus der Gruppe A kann vorzugsweise im BWS-Bereich die Reposition in der oben beschriebenen Weise indirekt durchgeführt und über die Instrumentierung ventral vervollständigt werden. Eine wesentliche Rolle spielt hierbei auch der Operationszeitpunkt. Erfahrungsgemäß gelingt eine anatomische Formwiederherstellung innerhalb der ersten 4 Tage nach Trauma vergleichsweise leicht, nach mehr als 1 Woche bringen die indirekten Repositionsmanöver häufig unbefriedigende Ergebnisse, so dass dann additiv dorsal zugegangen werden muss.

\section{Dekompression}

Die thorakoskopische Technik erlaubt eine komplette Dekompression des Spinalkanals unter Mitnahme aller notwendigen ligamentären und knöchernen Anteile. Die Sicht während der Resektion entspricht einem Operieren unter dem 

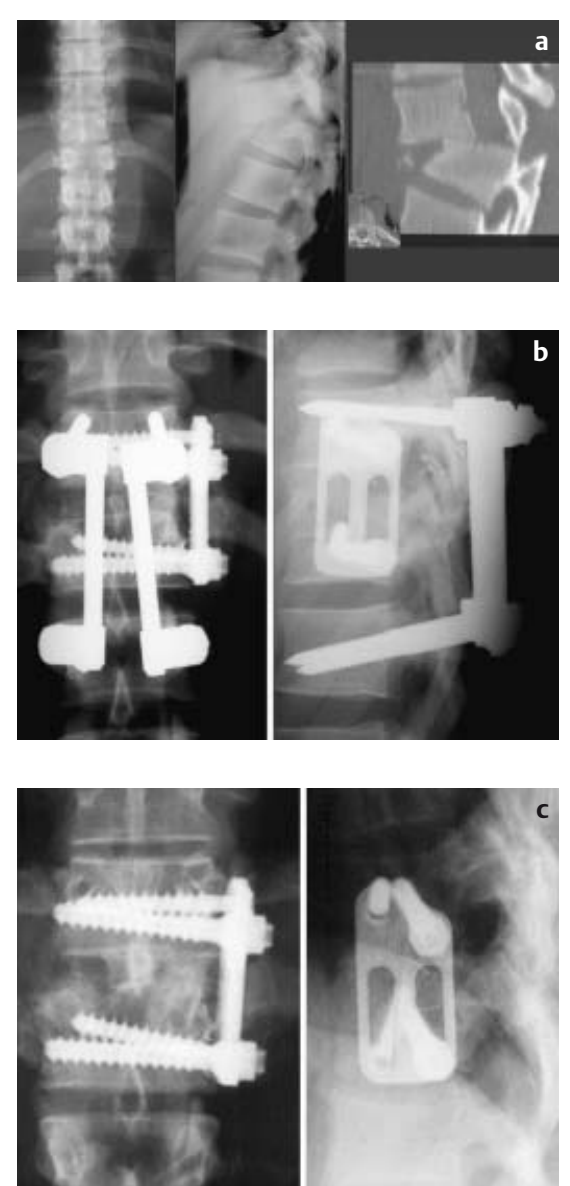

Abb.1a Luxationsfraktur des 12. Brustwirbelkörpers in der AO-Klassifikation Typ B 2.3. Patient mit kompletter Querschnittsymptomatik der Höhenlokalisation entsprechend.

Abb.1b Akutversorgung mittels dorsaler Reposition und Fixierung mit USS-Fixateur interne. 4 Tage später thorakoskopisch ventrale Fusion monsegmental zwischen 11. und 12. Brustwirbelkörper, Abstützung mit trikortikalem Beckenkammspan und zusätzlich winkelstabile Plattenosteosynthese.

Abb.1c Nach 5 Monaten Enfernung des Fixateur interne, verbliebene monosegmentale Spondylodese, die ventrale Instrumentation wird nicht entfernt.

Mikroskop. Die Operationstechnik wurde gegenüber dem offenen Vorgehen entsprechend modifiziert. Im ersten Operationsschnitt wird der knöcherne Wirbelbogenanteil an seinem Ansatz am Wirbelkörper durchtrennt und so die Dura zwischen zwei Nervenwurzelabgängen freigelegt. Anschließend erfolgt die Teilresektion des zerstörten Wirbelkörpers ventral im spongiösen Anteil. Im letzten Schritt wird dann die Wirbelkörperhinterkante quasi zwischen Dura und reseziertem zentralen Anteil freistehend abgetragen.
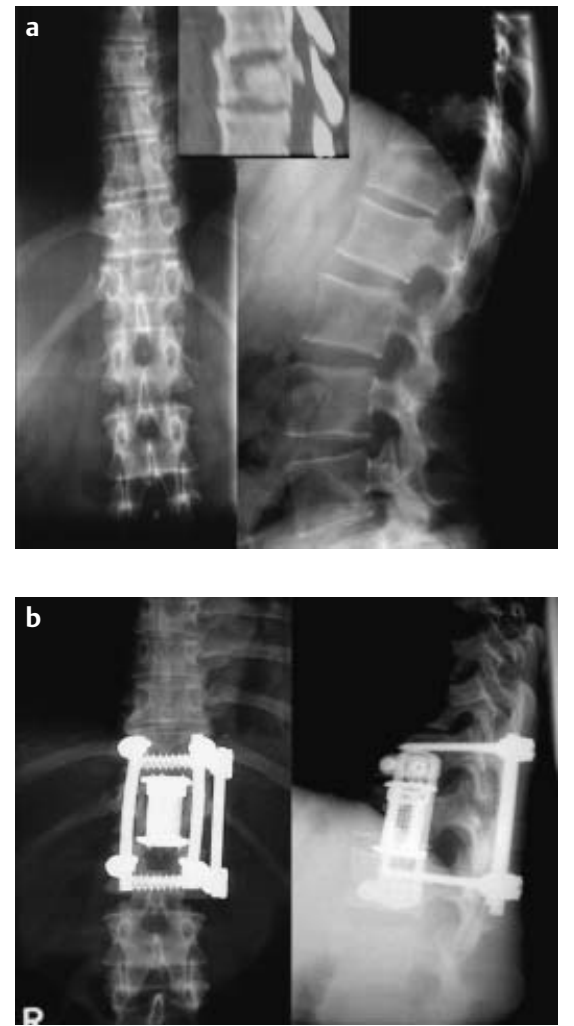

Abb. 2a Flektions-Distraktionsverletzung zwischen 11. und 12. Brustwirbelkörper. Berstungsfraktur des 12. Brustwirbelkörpers vom Kneifzangentyp. Verletzungsklassifikation nach AO-B 1.2.

Abb.2b Akutversorgung mit Aufrichtung und Reposition der Fraktur von dorsal mit USS-Fixateur interne. Nach 5 Tagen thorakoskopisch ventrale Resektion des 12. Brustwirbelkörpers mit Bandscheiben TH 11/TH 12 und TH 12/L 1. Zusätzlich Hinterkantenresektion und Fragmententfernung aus dem Spinalkanal. Wirbelkörperaufbau mit Synexkorb zwischen 11. Brustwirbelkörper und 1. Lendenwirbelkörper. Zusätzlich winkelstabile Plattenosteosynthese zwischen diesen Wirbelkörpern.

Die Indikation zur Dekompression anlässlich des thorakoskopischen Eingriffes wird anhand der CT-Kontrolle und dem klinischen Verlauf nach Akutversorgung gestellt. Im eigenen Vorgehen wird die wesentliche Dekompression des Spinalkanals meistens schon durch das Repositionsmanöver mittels Fixateur intern indirekt durchgeführt. Unter der Voraussetzung einer Akutversorgung gelingt dies am thorakolumbalen Übergang in über 90\% der Fälle. Liegen Einengungen durch die hinteren Wirbelstrukturen, z.B. dislozierte Fragmente der kleinen
Wirbelgelenke vor, wird eine Laminektomie durchgeführt. Verbleiben ventrale Fragmente im Spinalkanal, werden diese über die Laminektomie zurückgestößelt.

Die Indikation zur ventralen Dekompression anläßlich des thorakoskopischen Eingriffes wird bei einer relevanten verbliebenen Einengung des Spinalkanals gestellt. Weiterhin werden thorakoskopische Dekompressionen bei veralteten Fällen mit chronischer Spinalkanalenge oder entsprechender neurologischer Symptomatik durchgeführt.

\section{Rekonstruktion}

Für die Rekonstruktion der vorderen Säule bestehen in der Wirbelsäulentraumatologie divergierende Auffassungen.

Bei Anwendung rein dorsaler Versorgungsverfahren wird die transpedikuläre Spongiosaauffüllung der vorderen Säule empfohlen. Untersuchungen von Spätergebnissen durch verschiedene Studiengruppen haben gezeigt, dass diese Technik eine Sinterung bis hin zu einem ursprünglich bei der Fraktur bestandenen Dislokationsgrad effektiv nicht verhindern kann.

Diese Ergebnisse haben zahlreiche Kliniken zum Anlass genommen, die druckstabile Versorgung der vorderen Säule über einen zusätzlichen ventralen $\mathrm{Zu}$ gang durchzuführen. Zur Verwendung kommen dabei vorzugsweise Beckenkammspäne, die bikortikal oder solitär trikortikal eingebolzt werden. Im eigenen Vorgehen bevorzugen wir trikortikale autologe Späne vom vorderen Beckenkamm für die endoskopische Technik [8]. Zunehmend werden in geeigneten Fällen expandierbare Synexkörbe als Wirbelkörperersatz verwendet, die mit der aus dem Wirbelkörper resezierten Spongiosa umlegt werden (Abb.2).

\section{Retention}

Bei vorgehender dorsaler Instrumentierung mittels Fixateur intern kann dieser prinzipiell die Retention bis zur Einheilung der ventralen Rekonstruktionen übernehmen. Im eigenen Vorgehen führen wir jedoch regelmäßig auch eine ventrale Sicherung der Spondylodese mittels winkelstabiler Plattenosteosynthese durch. Die Vorteile dieses Vorgehens liegen zunächst einmal in der effektiven Protektion des ventralen Transplantates. 
Weiterhin kann ein Großteil der notfallmäßig zunächst bisegmental dorsal instrumentierten Verletzungen von ventral in monosegmentaler Ausdehnung fusioniert werden. Aufgrund der festen ventralen Spondylodese ist die Entfernung des Fixateur intern dann nach Ausheilung der dorsalen zugaufnehmenden Strukturen möglich. In der Regel erfolgt diese Entfernung nach 4 Monaten, so dass eine frühzeitige Remobilisierung des meist dorsal zunächst mitstabilisierten Segmentes möglich wird und der Sperreffekt des massiven dorsalen Implantates in der Rückenlängsmuskulatur beseitigt ist.

\section{Operatives Vorgehen}

Operiert wird in Seitenlage, am thorakolumbalen Übergang von links, bei Verletzungen oberhalb Th 8 vorzugsweise von rechts. Den ersten wesentlichen Operationsschritt stellt das Anzeichnen der Wirbelsäulenposition nach Bildwandlervisualisierung auf die Thoraxwand dar. Anhand der aufgezeichneten Lage der Wirbelkörper werden dann 4 Operationsportale eingezeichnet (Abb. 3). Das erste Portal wird in Minithorakotomietechnik in den Pleuraraum hinein eröffnet, um Verletzungen der Lungen bei allfälligen Verklebungen zu vermeiden. Nach Einbringen des Thorakoskopes über diesen Zugang wird die linke Lunge von der Anästhesie kontrolliert kollabiert, was durch Verwendung eines Doppellumentubus wesentlich erleichtert wird (Abb.4).

Im folgenden wird das Verletzungsareal aufgesucht und zunächst das dorsale Schraubenpaar in der geplanten Position eingebracht. Dieser Operationsschritt wird über Bildwandler kontrolliert. In der gesamten weiteren Folge der Operation dienen die dorsal definiert im Wirbelkörper liegenden Schrauben als optische Landmarken. Das Segmentgefäß über dem verletzten Wirbel wird dargestellt und zwischen Clips durchtrennt. Der Operationsraum innerhalb der Brusthöhle kann durch mehr oder weniger ausgedehntes Splitting des Zwerchfellmuskels bzw. des Psoas bis L3 ausgedehnt werden (Abb.5).

Entsprechend dem Resektionsausmaß wird die verletzte Bandscheibe mit dem Messer ausgelöst, die betroffene Wirbelkörperlateralwand mit dem Meißel ausgeschlagen. Die eigentliche Wirbelkörperresektion wird dann mit Stanzen und Fasszangen durchgeführt. Alle In-

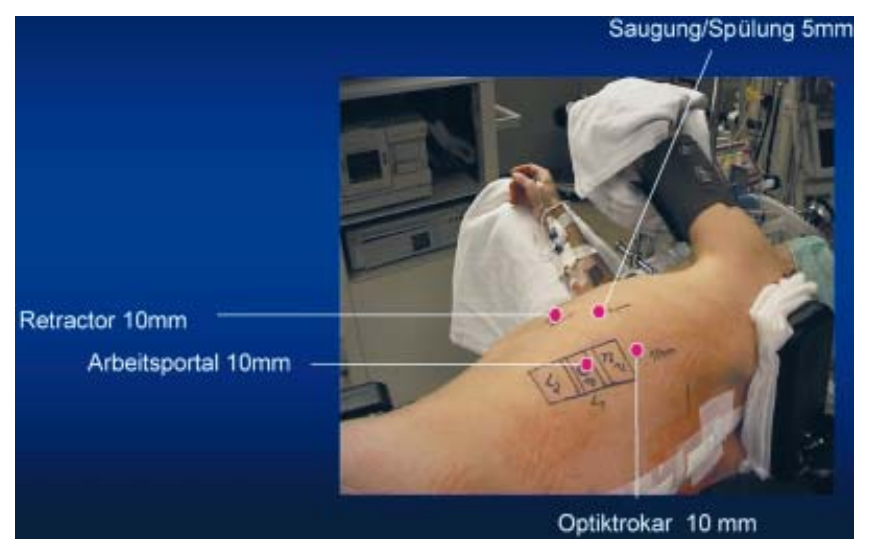

Abb. 3 Lagerung für eine thorakoskopisch kontrollierte Versorgung einer Fraktur des 1. Lendenwirbelkörpers. Rechte Seitenlage, die Wirbelsäule wird nach Lokalisierung mittels Bildwandler auf die Thoraxwand aufgezeichnet. Eingezeichnet sind die 4 für die endoskopische Versorgung notwendigen Portale.

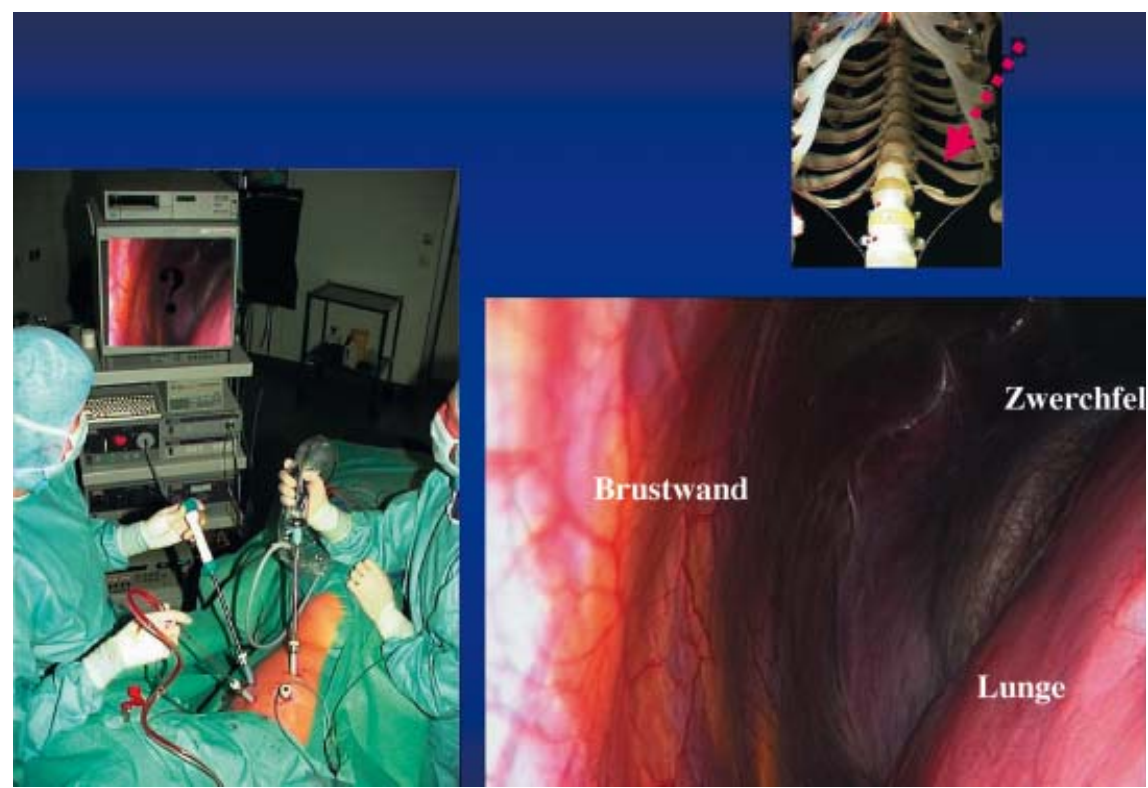

Abb. 4 Operativer Situs mit Operateur und Assistent, die sich gegenüberstehen. Auf dem Monitor ist der untere Recessus der Thoraxhöhle mit dem Zwerchfell abgebildet. Die Lunge ist teilkollabiert.

strumente weisen Skalierungen auf, die in der nicht räumlichen Darstellung der Videoskopie einen guten Anhalt zur Operationstiefe innerhalb des Wirbelkörpers geben. Die Wirbelkörperresektion kann ggf. als Hinterkantenresektion in der oben beschriebenen Technik ergänzt werden.

Nach Resektion fällt die Entscheidung zum Rekonstruktionsprinzip der vorderen Säule, die mittels Titankorbimplantation oder Einbringung eines trikortikalen Spans vorgenommen wird. Der Span wird in Press-Fit-Technik leicht konvergierend und mit leichter Übergröße eingebracht (Abb.6). Anschließend wird die Platte auf den dorsalen Schrauben aufgelegt, die ventralen Schrauben werden eingedreht und die endgültige Plattenspondylodese somit verwirklicht.
Nach Bildwandlerdokumentation in zwei Ebenen erfolgt der ggf. notwendige Verschluss des Zwerchfellsplitts mittels einzelner Stapler-Nähte oder fortlaufender Fadennaht. Eine Thoraxdrainage wird über eines der Portale eingelegt und platziert. Nach Rückzug der Instrumentierhülsen erfolgt der Nahtverschluss der Portale. Extubiert wird in der Regel im OP, der Pat. wird am nächsten Tag vor das Bett mobilisiert.

\section{Indikationen und zeitlicher Ablauf der Versorgung}

Bei allen Verletzungen der Brust- und Lendenwirbelsäule, die mit neurologischen Ausfällen einhergehen und eine hochgradige Instabilität entsprechend der AO-Klassifikation des B- und C-Typs aufweisen, sehen wir die Indikation zur notfallmäßigen oder zügigen dorsalen Instrumentierung. 


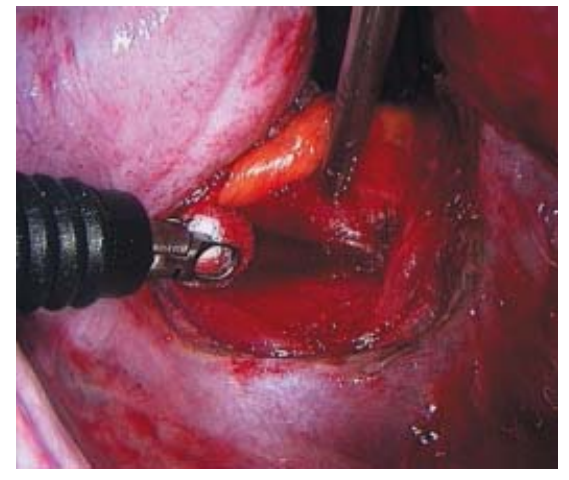

Abb. 5 Thorakoskopisches Bild des sogenannten Zwerchfellsplitting, der Stiltupfer führt eine Längsspaltung des unterhalb des Zwerchfells befindlichen Musculus psoas durch.
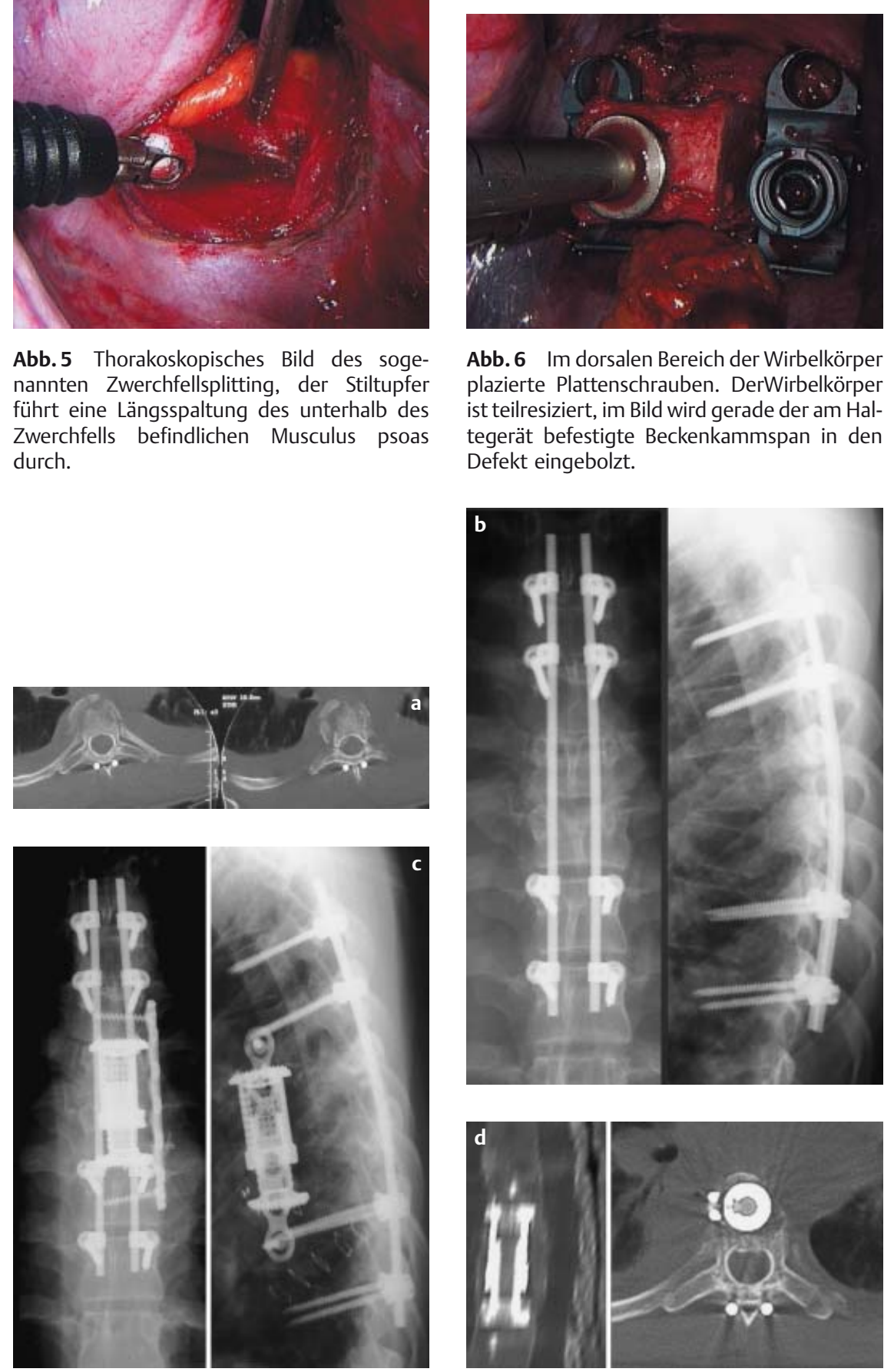

Abb. 6 Im dorsalen Bereich der Wirbelkörper plazierte Plattenschrauben. DerWirbelkörper ist teilresiziert, im Bild wird gerade der am Haltegerät befestigte Beckenkammspan in den Defekt eingebolzt.
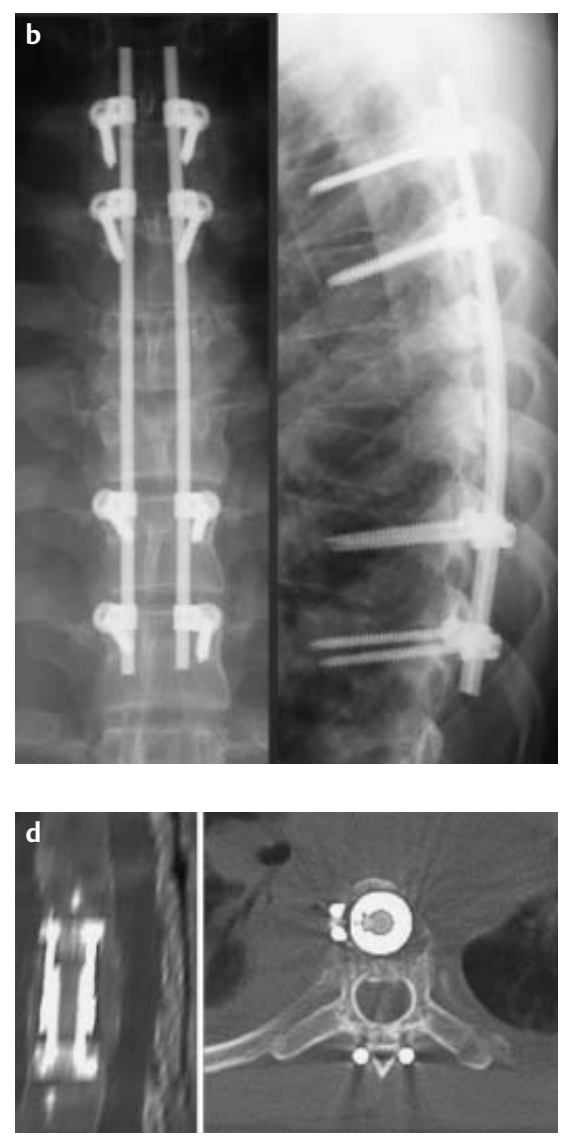

Abb. 7a Luxationsfraktur des 7. und 8. Brustwirbelkörpers mit kompletter Paraplegie auf dieser anatomischen Höhe.

Abb. 7b Akutversorgung langstreckig von BWK 5 bis BWK 10 über alleinigen dorsalen Zugang. Dadurch uneingeschränkte Lagerungstherapie auf der Intensivstation bei beidseitigen ausgedehnten Lungenkontusionen.

Abb.7c Bei ungestörtem Gasaustausch 2 Wochen nach Trauma ventrale thorakoskopisch gesteuerte Versorgung mit Resektion des 7. und 8. Brustwirbelkörpers und Ersatz durch Synex-Korb. Ventrale Zuggurtung durch einfache Plattenosteosynthese BWK 6 auf BWK 9.

Abb.7d Postoperative CT-Kontrolle bestätigt in der seitlichen Rekonstruktion die Wiederherstellung der Wirbelsäulenform, im Schnittbild die zentrale Lage des Synexkorbes.
Diese hat als Operationsziele die Reposition der Fehlstellung, damit meist verbunden die Dekompression des Spinalkanals sowie die vorläufige Retention der Fraktur.

Entsprechend dem Gesamtverletzungsausmaß, dem vorliegenden Verletzungstyp und der Organisationsplanung innerhalb der Klinik wird die ventrale Versorgung im Abstand von Tagen als Elektiveingriff durchgeführt. Bei schwerem Polytrauma, komplizierender Lungenkontusion oder anderen limitierenden Komplikationen ist naturgemäß die endgültige ventrale Versorgung auch mit einem größeren Zeitabstand ohne Nachteile möglich Abb. 7).

Im Intervall zwischen dorsaler und ventraler Versorgung wird eine Computertomographie durchgeführt, die Aufschluss über die korrekte Lage der Pedikelschrauben gibt und das Zerstörungsausmaß der vorderen Säule dokumentiert. Anhand dieser Aufnahmen lassen sich das notwendige Längenausmaß der ventralen Stabilisierung sowie die Dimension der zu verwendenden Schrauben abschätzen.

Geeignete Frakturkonstellationen entsprechend dem Typ A mit weitgehend intakt erhaltenen dorsalen Strukturen eignen sich für eine einzeitig rein ventrale Versorgung. Voraussetzung ist die Reponierbarkeit der Frakturstellung, die erfahrungsgemäß nur im frischen Stadium innerhalb von 4 Tagen befriedigend $\mathrm{zu}$ bewerkstelligen ist.

In Fehlstellung verheilte Verletzungskonstellationen mit Konsolidierung der knöchernen Komponenten müssen in der Regel kombiniert von dorsal und ventral angegangen werden.

Häufigstes Vorgehen ist die dorsale Mobilisierung der Strukturen mit transpedikulärer Instrumentation mittels Schanzschrauben, die folgende ventrale Mobilisierung mit dann kombinierter Reposition von dorsal und ventral. Anschließend erfolgt die Rekonstruktion der ventralen Säule mit anschließender Spondylodese wiederum ventral und dorsal. Für diese Operation ist in der Regel ein mehrmaliger Wechsel zwischen den operativen $\mathrm{Zu}$ gängen notwendig, die teilweise auch simultan bedient werden müssen. Auch für diese Situation hat sich die von uns bevorzugte Seitlagerung bewährt. 


\section{Ergebnisse}

Seit 1996 wurden mit der dargestellten endoskopischen Operationstechnik 300 Frakturen versorgt. Der Altersdurchschnitt der Patienten betrug 36 Jahre bei einer Spanne von 16-75 Jahren. Der Verletzungsschwerpunkt liegt erwartungsgemäß eindeutig im thorakolumbalen Übergang mit 2/3 der Verletzungen. Die Instabilitätseinschätzung zeigt zu $60 \%$ Verletzungen des Typs A.

2/3 der Patienten wurden im vorgestellten Sinne zweizeitig nach akuter Fixateur intern Stabilisierung operiert. Ein einzeitiger dorso-ventraler Kombinationseingriff erfolgte bei 7\% der Patienten, 31\% der Fälle, und somit $1 / 3$ wurden vorzugsweise im mittleren BWS-Bereich durch eine alleinige ventrale Spondylodese behandelt. Die Hälfte der Patienten erhielt ventral eine einsegmentale Fusion, die andere Hälfte 2- oder mehrsegmentale Spondylodesen.

Das Zwerchfellsplitting in der dargestellten Technik wurde bei der Hälfte der Patienten notwendig. Der intraoperative Blutverlust, ermittelt über das Volumen in der Zellsaver-Absaugung lag bei $520 \mathrm{ml}$. Über $1 / 3$ der Patienten wurde mit einem Blutverlust von unter $250 \mathrm{ml}$ operiert.

In den Operationszeiten spiegeln sich die technischen Probleme der Anfangszeit in Form einer Lernkurve wieder. Der anfängliche Zeitbedarf von durchschnittlich 4 bis 5 Stunden reduzierte sich mit durchschnittlicher Operationsdauer merklich und liegt inklusive Spangewinnung bei 3 Stunden derzeit. Die kürzeste monosegmentale Komplettversorgung erforderte bisher 70 Minuten.

In 3 Fällen war die Konversion zu einem offenen Eingriff notwendig, 2 dieser Fälle resultieren dabei aus der Anfangsphase der ersten 10 Fälle, bei einem Patienten konnte eine Schraubenverklemmung nicht endoskopisch behoben werden. In einem zweiten Fall kam es zu einer diffusen Blutung, die in diesem Stadium der operativen Erfahrung offen beherrscht wurde. Beim dritten Fall kam es bei einer schwierigen Revisionsoperation zu einer Verletzung der Aortenwand, die vorsichtshalber in offener Technik übernäht wurde.

Rezidivierende Pleuraergüsse wurden bei 18 Patienten gesehen und regelmäßig durch Punktion behandelt. Bei drei Pa- tienten wurde wegen Kammerung und zum Ausschluss eines Infektverdachtes eine endoskopische Spülung durchgeführt. Über neuralgieforme Beschwerden klagten 5 Patienten, andere postoperative neurologische Auffälligkeiten wie zwei Wurzelreizsyndrome mit Teilausfällen waren passager.

Zur Einschätzung der Beschwerdesymptomatik nach thorakoskopischem Eingriff wurden zwei 30-köpfige Patientengruppen mit vergleichbaren Versorgungsmustern nach offener und thorakoskopischer Intervention verglichen. Die Gesamtdosis der verabreichten Schmerzmittel war dabei nach endoskopischer Technik um $42 \%$ reduziert.

CT-Kontrollen wurden durchgehend bei allen Patienten durchgeführt. Fehllagen von Span und Platte mussten nicht beobachtet werden. Lediglich in zwei Fällen kam es zum Repositionsverlust, der einmal endoskopisch korrigiert wurde. Bei zwei Patienten erfolgte ein endoskopischer Schraubenwechsel wegen Lockerung. Spannekrosen zeigten sich bei zwei Patienten, in einem Fall wurde endoskopisch revidiert.

\section{Ausblick}

Eine Vielzahl technischer Probleme war für die Anfangsphase der thorakoskopisch assistierten Versorgung von Wirbelsäulenverletzungen kennzeichnend.

Inzwischen existieren geeignete Instrumente mit ausreichender Länge und speziellem Design für die endoskopische Technik.

Die Situseinstellung gelingt auch bei vorbestehenden pleuralen Verwachsungen regelmäßig. Das endoskopische Zwerchfellsplitting ist deutlich unproblematischer als die offene Technik mit teilweisem Abhängen des Zwerchfells. Verletzungen der gut sichtbaren großen Gefäße und des Herzens selbst sind prinzipiell nicht zu befürchten. Die einzige Aortenverletzung unserer Serie ereignete sich bei einer Revisionsoperation mit ausgeprägten narbigen Verwachsungen.

Die druckstabile Versorgung der ventralen Säule bleibt kontrovers. Die Interposition mit einem trikortikalen Beckenkammspan stellt nach heutigem Stand die biologisch optimale Lösung dar. Es bleibt allerdings das Problem der Morbidität des Spenderareals. Der Zugang zur
Gewinnung des Knochenspans bildet paradoxerweise den größten Hautschnitt während des ventralen Eingriffes. Die Beschwerdeangabe für die Entnahmestelle ist nicht zu vernachlässigen und übertrifft in der lokalen Schmerzhaftigkeit oft die eigentlichen operativen Zugänge. Weiterhin mussten wir für diese Region insgesamt fünf infektiöse Komplikationen beobachten, die allerdings unter programmierter Wundreinigung in versiegelter Vakuumtechnik sämtlich zur Abheilung gebracht werden konnten.

Ein alternatives überdenkenswertes Verfahren stellt die Implantation des auch endoskopisch einbringbaren Synexkorbes dar. In Verbindung mit einem Fixateur intern hat dieses Implantat wegen der sicher einzubringenden Vorspannung auch das Potenzial, die zusätzliche Plattenspondylodeze ventral entbehrlich $\mathrm{zu}$ machen.

Der Überblick während der thorakoskopischen Operation ist konkurrenzlos gut und übertrifft die Überschaubarkeit bei konventionell offenen und mehr noch bei sogenannten minimiert invasiven Eingriffen mittels speziellem Sperrer in eindrucksvoller Weise.

Dies stellt einen erheblichen Vorteil für die Sicherung der operativen Qualität und die Möglichkeiten zusätzlicher Maßnahmen an benachbarten Segmenten dar. Die Resektion der Hinterkante und das operative Vorgehen im Spinalkanal erreichen die Qualität eines Operationsmikroskops und können mit entsprechender Präzision durchgeführt werden. Entsprechend den Erfahrungen, z.B. mit der Arthroskopie, lässt sich daher unschwer vorhersagen, dass sich die Operation unter thorakoskopischen Bedingungen sehr rasch durchsetzen wird. Für die zunehmend häufiger induzierte Metastasenchirurgie bietet die endoskopische Technik eine dem Allgemeinzustand angepasste schonende Alternative.

Parallel zu den Entwicklungen in der Chirurgie der thorakalen Organe ist $\mathrm{zu}$ schlussfolgern, dass die thorakoskopische Versorgung der Brust- und Lendenwirbelsäule die offenen Verfahren in relativ kurzer Zeit zum größten Teil ersetzen wird. An der prinzipiellen Machbarkeit bestehen keinerlei Zweifel. Der Zeitbedarf gleicht sich mit zunehmender Übung den offenen Verfahren an, wie dies im übrigen auch aus den Erfahrungen der arthroskopischen und laparoskopischen Operationen prognostiziert werden kann. 


\section{Literatur}

${ }^{1}$ Beisse R, Potulski M, Temme C, Bühren V. Das endoskopisch kontrollierte Zwerchfellsplitting - ein minimal-invasiver Zugang zur ventralen Versorgung thorakolumbaler Frakturen der Wirbelsäule. Unfallchirurg 1998; 101: 619-627

${ }^{2}$ Beisse R, Potulski M, Bühren V. Thorakoskopisch gesteuerte ventrale Plattenspondylodese bei Frakturen der Brust- und Lendenwirbelsäule. Operat Orthop Traumatol 1999; 11: 54-69

${ }^{3}$ Beisse R, Potulski M, Bühren V. Thoracoscopic-Assisted Anterior Approach to Thoracolumbar Fractures. In: Mayer HM. Minimally Invasive Spine Surgery, Springer, Berlin, Heidelberg, New York 2000; S. 175-186

${ }^{4}$ Bühren V, Braun Ch. Ventrale Fusionsosteosynthese bei Frakturen der Brustwirbelsäule. Oper Orhop Traumatol 1993; 5: 245-258
${ }^{5}$ Bühren V, Beisse R, Potulski M. Minimalinvasive ventrale Spondylodesen bei Verletzungen der Brust- und Lendenwirbelsäule. Chirurg 1997; 68: 1076-1084

${ }^{6}$ Jacobaeus HC. Über die Möglichkeit, die Zystoskopie bei der Untersuchung seröse Höhlen anzuwenden. Münch Med Wochenschr 1910; 57: 2090

${ }^{7}$ Mack MJ, Regan J, Bobechko WP, Acuff TE Application of thoracoscopy for diseases of spine. Ann Thorac Surg 1993; 56: 736-738

8 Potulski M, Beisse R, Bühren V. Die thorakoskopisch gesteuerte Behandlung der „vorderen Säule“ - Technik und Ergebnisse Orthopäde 1999; 28: 723-730

${ }^{9}$ Regan JJ, Mc Afee P, Mack M. Atlas of endoscopic spine surgery. Quality Medical, Publishing, St. Louis 1995

${ }^{10}$ Trentz O, Friedl HP. Brust- und Lendenwirbelsäule. In: Rüter A, Trentz $\mathrm{O}$, Wagner $\mathrm{M}$ Unfallchirurgie. Urban und Schwarzenberg München Wien Baltimore 1995; S. 421 - 451
Prof. Dr. med. V. Bühren

Ärztlicher Direktor

Dr. med. R. Beisse

Leitender Arzt

Dr. med. M. Potulski

Leitender Arzt

BG-Unfallklinik

Professor-Küntscher-Str. 8

82418 Murnau 